\title{
Derivação gástrica em Y-de-Roux e a atividade inflamatória do tecido adiposo
}

\section{Roux-en-Y gastric bypass and inflammatory activity of the adipose tissue}

\author{
Antonio Carlos Valezi, TCBC-PR; Edilson João Cabrera 2 ; Vinicius Daher Alvares Delfino33; Decio Sabbatini Barbosa4; \\ Jorge Mall Junior, TCBC-PR ${ }^{5}$; Mariano de Almeida Menezes ${ }^{6}$
}

\section{R E S U M O}

\begin{abstract}
Objetivo: Avaliar os indicadores de estresse oxidativo e marcadores inflamatórios em obesos antes e depois da gastroplastia vertical com bandagem em Y-de-Roux. Métodos: Vinte obesos classe III foram submetidos à gastroplastia vertical com bandagem em Y-deRoux, sendo sete homens e 13 mulheres, com idade média de 39 anos e 20 indivíduos não obesos, nove homens e 11 mulheres, média de idade de 38 anos. Foram determinados os níveis de malondialdeído no plasma, índice de estresse, capacidade antioxidante total, catalase e glutationa reduzida e oxidada, e marcadores inflamatórios (proteína C reativa e á-1 glicoproteína ácida). No grupo obeso, estes parâmetros foram determinados antes e 2, 6 e 12 meses após a gastroplastia vertical com bandagem em Y-de-Roux. Resultados: No pré-operatório, o grupo obeso apresentou níveis elevados de marcadores inflamatórios, de estresse oxidativo (níveis de malondialdeído e índice de estresse) e menores níveis de indicadores de defesa antioxidante em relação ao grupo controle. O emagrecimento foi acompanhado de redução progressiva do níveis de malondialdeído e do índice de estresse. Foi observado aumento da concentração de glutationa reduzida e da capacidade antioxidante total e redução dos níveis de marcadores inflamatórios. Conclusão: A redução do peso melhora o estado inflamatório e os níveis de estresse oxidativo.
\end{abstract}

Descritores: Cirurgia Bariátrica. Estresse Oxidativo. Obesidade. Marcadores Biológicos.

\section{INTRODUÇÃO}

Indivíduos obesos apresentam elevados níveis circulantes de citocinas inflamatórias como a interleucina 6 (IL6) e o fator de necrose tumoral alfa (TNF $\alpha)$, ambas produzidas pelo tecido adiposo'. Estas citocinas ativam células (fagócitos, fibroblastos, linfócitos B e células endoteliais) que vão liberar moléculas pró-oxidantes, principalmente as espécies reativas de oxigênio - EROs².

Praticamente todas as biomoléculas (proteínas, lipídeos e DNA) são suscetíveis à oxidação e, para se proteger, o organismo possui mecanismos químicos e enzimáticos com propriedades antioxidantes ${ }^{3}$. Nas situações em que a proteção é insuficiente e/ou há excesso de substâncias pró-oxidantes, ocorre estresse oxidativo ${ }^{4}$.

A redução do tecido adiposo diminui a secreção de citocinas inflamatórias e consequente queda no nível de estresse oxidativo, diminuindo os fatores de risco para as doenças relacionadas à obesidade ${ }^{5}$.

Neste estudo avaliaram-se marcadores inflamatórios, indicadores de estresse oxidativo e da defesa antioxidante em indivíduos obesos classe III, submetidos à gastroplastia vertical com bandagem em Y-de-Roux (GVBYR). Monitoraram-se estes parâmetros no pré-operatório, 2, 6 e 12 meses após a operação.

\section{MÉTODOS}

Este estudo está em consonância com a resolução 196/96 do Conselho Nacional de Saúde/Comissão Nacional de Ética em Pesquisa, tendo sido aprovado pelo Comitê de Ética em Pesquisa em Seres Humanos da Universidade Estadual de Londrina (Parecer CEP 041/05).

Os participantes foram distribuídos em dois grupos: grupo obeso e grupo controle. O grupo inicial de obesos era composto por 25 indivíduos. Deste grupo foram excluídos cinco indivíduos: um apresentou infecção póscirúrgica, um necessitou de nova operação e três não compareceram para efetuar a coleta de amostra nos períodos determinados. No grupo de não obesos (controle), foram excluídos dois indivíduos por apresentarem alteração nos parâmetros inflamatórios analisados.

Trabalho realizado no Hospital Universitário da Universidade Estadual de Londrina, Londrina, PR, Brasil.

1. Professor Associado do Departamento de Cirurgia da Universidade Estadual de Londrina - PR-BR; 2. Farmacêutico do Hospital Universitário da Universidade Estadual de Londrina- PR-BR; 3. Professor do Departamento de Clínica Médica da Universidade Estadual de Londrina- PR-BR; 4. Professor do Departamento de Patologia, Análises Clínicas e Toxicologia da Universidade Estadual de Londrina- PR-BR; 5. Cirurgião do Hospital Universitário da Universidade Estadual de Londrina - PR-BR; 6. Pós Graduando do Centro de Ciências da Saúde da Universidade Estadual de Londrina- PR-BR. 
Os grupos participantes do estudo ficaram assim constituídos: grupo obeso composto por 20 pacientes, sendo sete homens e 13 mulheres, com idade entre 26 e 52 anos e o grupo controle composto por 20 indivíduos não obesos sendo 9 homens e 11 mulheres com idade entre 27 e 53 anos, estes não estavam sob nenhum tipo de tratamento médico ou em uso de medicamentos.

A coleta de amostras para dosagem de marcadores de estresse oxidativo no grupo de obesos foi feita no período pré-operatório, 2, 6 e 12 meses após a operação. A coleta de amostras dos 20 indivíduos que formam o grupo controle (não obeso) foi feita em apenas um momento.

Como indicador de oxidação utilizou-se a concentração plasmática do dialdeído malônico (MDA), um subproduto da lipoperoxidação. Para avaliação da defesa antioxidante, utilizou-se as enzimas SOD (superóxido dismutase), catalase, glutationa total e oxidada, e avaliouse a capacidade antioxidante total pelo TRAP (Total radical antioxidant parameter) plasmático. O protocolo deste estudo incluiu a dosagem de parâmetros inflamatórios (proteína C Reativa - PCR e alfa-1 glicoproteína ácida - GPA). Todos os exames foram realizados em duplicata, aceitando-se o coeficiente máximo de variação de 10\% em torno da média.

Para o cálculo do índice de estresse (IE), que indica o estado de oxidação nas hemácias, utilizaram-se os níveis de glutationa, segundo a fórmula:

$\mathrm{IE}=$ GSSG (glutationa oxidada)/GSH (glutationa reduzida) - GSSG

Os resultados foram expressos por média e desvio-padrão e apresentados em forma de tabelas e figuras. Foi realizado o teste de Shapiro-Wilk para testar a normalidade dos achados. Na comparação entre os grupos foi utilizado o teste t de Student para amostras não pareadas. Para verificar a variância intra grupo foi utilizado o teste de Anova com post hoc test de Bonferroni quando necessário. A análise de correlação foi feita pelo teste de correlação de Pearson.

Diferenças significativas foram determinadas por $p<0,05$ e correlações importantes (quando $r>0,45$ ), foram selecionadas para análise. Foi utilizado o software SPSS (Statistical Package for the Social Sciences) para o Windows, versão 13, 0, para análise dos dados.

\section{RESULTADOS}

Os valores médios do marcador inflamatório PCR do grupo de obesos no período pré-operatório estavam elevados em relação ao do grupo controle $(p<0,001)$ e sofreu redução gradativa, sendo esta estatisticamente significativa entre todos os momentos avaliados. Decorridos 12 meses da operação, estes valores já não apresentavam diferença em relação ao grupo controle. A GPA apresentou comportamento semelhante à $P C R$, com redução dos valores médios no grupo obeso em todos os momentos avaliados, e valores no período pré-operatório maiores que no grupo controle $(p<0,001)$ (Tabela 1).

Em relação à avaliação das defesas antioxidantes, a catalase não apresentou diferença entre os grupos controle e obeso no período pré-operatório e entre os diferentes momentos avaliados. A GSH e o TRAP, que apresentavam valores diminuídos no grupo de obesos no período pré-operatório em relação ao grupo controle $(p<0,001)$, mostraram aumento significativo após a operação, até que, passados 12 meses, já não havia diferenças significativas em relação ao grupo controle. A SOD apresentou atividade maior no grupo obeso no período pré-operatório em relação ao grupo controle $(p<0,001)$ e essa atividade diminuiu ao longo dos períodos avaliados; mas, aos 12 meses, permanecia elevada em relação ao grupo controle $(p<0,001)$. Os dados estão apresentados na tabela 2.

Os marcadores de lesão oxidativa (MDA e IE) estavam significativamente aumentados $(p<0,001)$ no grupo obeso no período pré-operatório em relação ao grupo controle. O MDA plasmático apresentou queda estatisticamente significativa entre todos os períodos avaliados do grupo obeso. Decorridos 12 meses após a operação, os níveis médios ainda estavam significativamente maiores $(p<0,001)$ do que aqueles encontrados no grupo controle. Quanto ao MDA, embora tenha-se observado significativa redução dos valores no grupo obeso, após 12 meses da operação, estes valores mantinham-se elevados em relação ao grupo controle $(p<0,01)$. O IE estava elevado no grupo obeso no período pré-operatório em relação ao grupo controle e, após a operação, mostrou redução significativa no

Tabela 1 - Resultados da dosagem de proteína C reativa e $\alpha$-1 glicoproteína ácida.

\begin{tabular}{|c|c|c|c|c|c|c|}
\hline \multirow[t]{2}{*}{ TESTES } & Controle (a) & Pré-op (b) & 2 meses $(c)$ & 6 meses $(d)$ & 12 meses (e) & \multirow{2}{*}{$\begin{array}{l}\text { Valor de } p \\
\text { entre grupos }\end{array}$} \\
\hline & $\begin{array}{l}\text { Grupo controle } \\
(n=20)\end{array}$ & $\begin{array}{l}\text { Grupo obeso } \\
(n=20)\end{array}$ & $\begin{array}{l}\text { Grupo obeso } \\
(n=20)\end{array}$ & $\begin{array}{c}\text { Grupo obeso } \\
(n=20)\end{array}$ & $\begin{array}{l}\text { Grupo obeso } \\
\quad(n=20)\end{array}$ & \\
\hline $\mathrm{PCR}(\mathrm{mg} / \mathrm{dL})$ & $0,48 \pm 0,24$ & $1,30 \pm 0,97$ & $0,93 \pm 0,42$ & $0,64 \pm 0,40$ & $0,38 \pm 0,26$ & $\begin{array}{c}* b / c^{* *} c / e^{* * *} a / b \\
b / d, b / e\end{array}$ \\
\hline $\mathrm{GPA}(\mathrm{mg} / \mathrm{dL})$ & $58 \pm$ & $126 \pm$ & $108 \pm 34$ & $84 \pm 24$ & $76 \pm$ & $\begin{array}{c}* c / d^{* *} c / e{ }^{* * *} a / b, \\
a / e, b / d, b / e\end{array}$ \\
\hline
\end{tabular}

Pré-op $=$ Pré-operatório. ${ }^{*} p<0,05 .{ }^{* *} p<0,01 .{ }^{*}{ }^{*} p<0,001 . P C R=$ proteína $C$ reativa. GPA= $\alpha-1$ glicoproteína ácida. 
grupo obeso entre todos os períodos avaliados. Neste grupo, no período de 12 meses após a operação, os valores médios ainda estavam elevados em relação aos do grupo controle $(p<0,001)$. Os dados estão apresentados na tabela 3.

\section{DISCUSSÃO}

A relação entre perda de peso e redução dos níveis de PCR já foi demonstrada ${ }^{6}$. A síntese de PCR hepática é estimulada principalmente pela IL-6 e o adipócito produz em torno de $30 \%$ da IL-6 circulante de origem não inflamatória ${ }^{7}$. A produção de IL-6 está diretamente relacionada ao volume do tecido adiposo, e a perda de peso causada pela GVBYR faz com que haja menor quantidade de IL-6 circulante com consequente queda da síntese hepática da $P C R$, reduzindo os efeitos biológicos desta proteína. Entre estes efeitos estão sua ação direta sobre o endotélio vascular induzindo à síntese de moléculas de adesão $^{8}$ e ao aumento da geração e liberação de EROs pelos neutrófilos ${ }^{9}$.
Ainda que a função biológica da GPA permaneça desconhecida, é um indicador que se altera na presença de processos que causam agressão tecidual (inflamação ou infecção) e sua síntese hepática é regulada por citocinas como a IL-1, IL-6, outras citocinas da família da IL-6 (como a leptina) e TNF-á10. Os estudos envolvendo GPA como marcador inflamatório mostram que ela tem sido mais utilizada no acompanhamento e avaliação de recorrências em pacientes com neoplasias ${ }^{11}$

Quanto aos marcadores inflamatórios PCR e GPA, este estudo demonstrou diferença estatisticamente significativa entre os pacientes do grupo controle e do grupo obeso no período pré-operatório. Dois meses após a operação, verificou-se redução dos níveis de PCR e de GPA e correlação positiva entre estes indicadores $(r=0,837$ e pd"0,001). A redução nos níveis de GPA não foi significativa neste período, ao passo que a PCR já apresentou variação significativa, provavelmente devido a esta proteína ser o mais precoce e sensível indicador de processo inflamatório ${ }^{12}$. As avaliações nos períodos seguintes mostraram redução gradativa dos valores médios da PCR e da GPA no grupo obeso.

Tabela 2 - Marcadores de defesa antioxidante.

\begin{tabular}{|c|c|c|c|c|c|c|c|}
\hline \multirow[t]{2}{*}{ TESTES } & \multicolumn{2}{|c|}{ Controle (a) } & Pré-op (b) & 2 meses $(c)$ & 6 meses $(d)$ & 12 meses (e) & \multirow{2}{*}{$\begin{array}{l}\text { Valor de } p \\
\text { entre grupos }\end{array}$} \\
\hline & $\begin{array}{l}\text { Grupo } \\
\qquad(\mathrm{n}=\end{array}$ & $\begin{array}{l}\text { controle } \\
20)\end{array}$ & $\begin{array}{c}\text { e Grupo obeso } \\
(n=20)\end{array}$ & $\begin{array}{c}\text { Grupo obeso } \\
(n=20)\end{array}$ & $\begin{array}{l}\text { Grupo obeso } \\
\qquad(n=20)\end{array}$ & $\begin{array}{l}\text { Grupo obeso } \\
(n=20)\end{array}$ & \\
\hline $\begin{array}{l}\text { CATALASE } \\
(\triangle / \mathrm{mgHb} / \mathrm{min})\end{array}$ & n) $12,3 \pm$ & 1,26 & $12,1 \pm 1,19$ & $12,7 \pm 1,20$ & $12,8 \pm 1,30$ & $13,2 \pm 1,56$ & NS \\
\hline $\begin{array}{l}\text { SOD } \\
(\mathrm{U} / \mathrm{mgHb})\end{array}$ & $7,56 \pm$ & $\pm 0,79$ & $10,70 \pm 1,78$ & $10,34 \pm 1,10$ & $9,74 \pm 1,10$ & $9,24 \pm 1,27$ & $\begin{array}{c}{ }^{*} \mathrm{~b} / \mathrm{d}, \mathrm{c} / \mathrm{d}^{* *} \mathrm{c} / \mathrm{e}^{* * *} \mathrm{a} / \mathrm{b}, \\
\mathrm{a} / \mathrm{e} \text { e } \mathrm{b} / \mathrm{e}\end{array}$ \\
\hline $\begin{array}{l}\mathrm{GSH} \\
(\mathrm{mM} / \mathrm{g} \text { de } \mathrm{Hb})\end{array}$ & $2728 \pm 3$ & 315 & $2002 \pm 249$ & $2276 \pm 282$ & $2598 \pm 314$ & $2833 \pm 249$ & $\begin{array}{c}{ }^{* *} \mathrm{~d} / \mathrm{e}^{* * *} \mathrm{a} / \mathrm{b}, \mathrm{b} / \mathrm{c}, \mathrm{b} / \mathrm{d} \\
\mathrm{b} / \mathrm{e}, \mathrm{c} / \mathrm{d} \text { e c/e }\end{array}$ \\
\hline $\begin{array}{l}\text { GSSG } \\
(\mathrm{mM} / \mathrm{g} \text { de } \mathrm{Hb})\end{array}$ & $127 \pm$ & 14 & $211 \pm 35$ & $181 \pm 28$ & $160 \pm 24$ & $148 \pm$ & $\begin{array}{c}{ }^{*} c / e^{* * *} a / b, a / e, b / c \\
b / d, b / e ~ e ~ c / e\end{array}$ \\
\hline $\begin{array}{l}\text { TRAP (plasma) } \\
\left(\square \mathrm{M} \text { Trolox }^{\circledR}\right)\end{array}$ & $760,7 \pm$ & 64,3 & $585,5 \pm 148,4$ & $615,2 \pm 111,6$ & $717,5 \pm 124,5$ & $815,5 \pm 149,4$ & $\begin{array}{c}* * b / d, c / d * * * a / b \\
b / e \text { e c/e }\end{array}$ \\
\hline
\end{tabular}

Pré-op= Pré-operatório * $p<0,05 \quad * * p<0,01 \quad * * * p<0,001$

$S O D=$ Superóxido dismutase, GSH= Glutationa reduzida, GSSG= Glutationa oxidada, TRAP (Total Radical Antioxidant Parameter)= Capacidade antioxidante total

Tabela 3 - Indicadores de lesão oxidativa.

\begin{tabular}{|c|c|c|c|c|c|c|}
\hline \multirow[t]{2}{*}{ TESTES } & Controle (a) & Pré-op (b) & 2 meses $(c)$ & 6 meses $(d)$ & 12 meses (e) & \multirow{2}{*}{$\begin{array}{l}\text { Valor de } p \\
\text { entre grupos }\end{array}$} \\
\hline & $\begin{array}{l}\text { Grupo controle } \\
\qquad(n=20)\end{array}$ & $\begin{array}{l}\text { Grupo obeso } \\
\qquad(n=20)\end{array}$ & $\begin{array}{l}\text { Grupo obeso } \\
\qquad(n=20)\end{array}$ & $\begin{array}{l}\text { Grupo obeso } \\
\qquad(n=20)\end{array}$ & $\begin{array}{l}\text { Grupo obeso } \\
\qquad(n=20)\end{array}$ & \\
\hline IE & $0,049 \pm 0,05$ & $0,121 \pm 0,03$ & $0,091 \pm 0,02$ & $0,069 \pm 0,02$ & $0,055 \pm 0,06$ & $\begin{array}{c}{ }^{*} \mathrm{~d} / \mathrm{e}^{* * *} \mathrm{a} / \mathrm{b}, \mathrm{a} / \mathrm{e}, \\
\mathrm{b} / \mathrm{c}, \mathrm{b} / \mathrm{d}, \mathrm{b} / \mathrm{e}, \mathrm{c} / \mathrm{d} \text { e } \mathrm{e} / \mathrm{e}\end{array}$ \\
\hline $\begin{array}{l}\text { MDA (plasma) } \\
\text { (nmol/g prot) }\end{array}$ & $4,99 \pm 1,19$ & $16,69 \pm 8,23$ & $15,04 \pm 8,10$ & $11,61 \pm 5,45$ & $9,10 \pm 4,19$ & $\begin{array}{l}* * b / d, c / e^{* \star \star} \\
\text { a/b e a/e e b/e }\end{array}$ \\
\hline
\end{tabular}

Pré-op $=$ Pré-Operatório $\quad * p<0,05 \quad * * p<0,01$

$M D A=$ dialdeído malônico, $I E=$ índice de estresse. 
Os níveis de GPA apresentaram correlação positiva e significante com os níveis de PCR em obesos no período pré-operatório $(r=0,809, p<0,01)$ e 12 meses após a operação $(r=0,579$ e $p<0,01)$, provavelmente como reflexo da queda dos níveis de citocinas em função da redução do tecido adiposo após GVBYR, sugerindo a possibilidade de utilização deste marcador como indicador do estado inflamatório em pacientes obesos.

Os pacientes participantes do estudo receberam suplemento vitamínico a partir de 60 dias após a operação, por meio da administração de um comprimido diário do polivitamínico Centrum ${ }^{\circledR}$ que inclui alguns antioxidantes como a vitamina A (5.000 UI), vitamina E (30 UI) e a vitamina C (60 mg). O papel destes antioxidantes e sua relação com as diversas doenças têm sido descritos por vários autores ${ }^{13}$. A grande diferença entre dosagens, metodologias, grupos de estudo e resultados obtidos não nos permite concluir sobre o efeito que estes antioxidantes podem exercer sobre o nível de estresse oxidativo em pacientes portadores de doenças crônicas. Visto que a administração de polivitamínico faz parte da terapêutica pós-cirúrgica da obesidade, sua influência sobre os resultados obtidos neste estudo soma-se à perda do peso e às alterações na dieta destes pacientes.

Não foram observadas alterações significativas nos níveis dos indicadores de lipoperoxidação (MDA plasmático) e da atividade das enzimas antioxidantes SOD e catalase. O TRAP apresentou melhora sem significância estatística. Quanto à glutationa, observou-se redução nos níveis de GSSG, aumento da GSH, redução no índice de estresse e correlação inversa entre os níveis de glutationa oxidada e o TRAP $(r=-0,484, p<0,05)$, mostrando que quanto maior o nível de TRAP, menor quantidade de glutationa oxidada. Estas observações refletem melhora da defesa antioxidante neste período.

A associação entre níveis elevados de estresse oxidativo e obesidade foi estabelecida em diversos trabaIhos, envolvendo modelos animais ${ }^{14}$ e humanos ${ }^{15}$. Entre as biomoléculas que sofrem ação nociva de radicais livres, os lipídeos são a principal classe envolvida. O processo de oxidação de lipídeos é chamado de lipoperoxidação (LPO) e pode ocorrer na membrana celular, nos ácidos graxos livres ou nas lipoproteínas como o LDL-colesterol ${ }^{16}$. Entre os subprodutos gerados pela LPO, está o malondialdeído (MDA) que pode ser usado como marcador de lesão oxidativa ${ }^{17}$. Além desse papel, o MDA possui ação tóxica sobre o DNA. Apesar de sua baixa reatividade em pH fisiológico, esta molécula é capaz de reagir com ácidos nucléicos, alterando o DNA podendo estar relacionada ao desenvolvimento de vários tipos de câncer ${ }^{18}$. Neste estudo realizou-se a dosagem do MDA no plasma como indicador de oxidação de lipídeos de membrana, ácidos graxos livres ou lipoproteínas. Os níveis plasmáticos médios de MDA no grupo obeso, no período pré-operatório, estavam significativamente elevados em relação aos encontrados em indivíduos do grupo controle mostrando que os indivíduos obesos apresentam maior teor de oxidação das lipoproteínas.

O emagrecimento é acompanhado por redução na geração de $\mathrm{EROs}^{3}$ e queda nos níveis de $\mathrm{MDA}^{19}$. Em concordância com estes dados, observou-se, após a operação, redução significativa dos níveis médios de MDA plasmático que estavam significativamente elevados no grupo obeso em relação ao grupo controle no período préoperatório e sofreram redução significativa em seus valores refletindo queda nos índices de oxidação da fração LDLcolesterol.

Para avaliação do perfil das defesas antioxidantes, analisou-se a atividade das enzimas SOD, catalase, os níveis da glutationa reduzida e oxidada e o TRAP. Encontrou-se maior atividade da SOD no grupo obeso em relação ao grupo controle, mesmo 12 meses após a operação. Embora tenha ocorrido redução na atividade pósoperatória da SOD, estes níveis permaneceram elevados e apresentaram diferença em relação ao grupo controle em todos os períodos avaliados. No grupo obeso, observou-se correlação positiva entre o peso e a SOD $(r=0,444$, $p<0,05)$ no período pré-operatório que não foi observada 12 meses após a operação. Uma maior atividade de SOD no indivíduo obeso está relacionada a aumento das condições que causam indução da síntese da SOD como o TNF-á20 e a IL$6^{21}$, ou seja, a presença de inflamação crônica e estresse oxidativo na obesidade podem resultar em aumento da geração de radicais superóxido, ocasionando resposta adaptativa com consequente aumento da atividade da SOD. A perda do peso aliada a menor grau de síntese e liberação de EROs e citocinas, possivelmente, apresentou redução no efeito indutor sobre a SOD, o que explicaria essa ausência de correlação 12 meses após a operação.

Estes dados demonstraram que não houve alteração pós-operatória da atividade da catalase no grupo obeso, e que a atividade da catalase era semelhante no grupo de pacientes obesos em relação ao grupo controle no período pré-operatório. Dados de estudos prévios demonstraram que a atividade da catalase apresenta grande variação de atividade. Nageswara et al. ${ }^{22}$ não observaram alteração da catalase ao avaliar a hipertensão associada aos componentes da síndrome metabólica. Estudos in vitro indicaram que o aumento dos níveis de citocinas inibe a atividade da catalase em células beta ${ }^{23}$ e que um baixo grau de oxidação pode induzir resposta adaptativa, aumentando a atividade desta enzima antioxidante em células vasculares $^{24}$. Estas diferenças entre vários estudos podem ser parcialmente explicadas em função dos diversos modelos experimentais e intervenções utilizadas, bem como por variações no sexo, idade, grau e tempo de duração da doença em que a atividade da catalase foi avaliada.

A glutationa existe no organismo humano em duas formas: uma reduzida e outra oxidada. Utilizou-se o valor da glutationa reduzida (GSH) e da oxidada (GSSG) para calcular o índice de estresse (IE), pois esta relação pode ser um bom marcador biológico de estresse oxidativo ${ }^{25}$. 
Entre os pacientes obesos, encontrou-se correlação inversa entre os níveis de GSH e o IE $(r=-0,598, p<0,01)$, e correlação direta do GSSG com o IE no período pré-operatório $(r=0,778, p<0,01)$. Doze meses após a operação estas correlações persistiam $(r=-0,671, p<0,01$ e $r=0,541, p<0,05)$. Os pacientes do grupo obeso apresentaram menor nível médio de GSH no período pré-operatório em comparação com o grupo controle, mas 12 meses do pós-operatório, estes níveis já não apresentaram diferença significativa. Os valores do IE sofreram queda significativa em todos os períodos avaliados no grupo obeso. A queda dos níveis de glutationa reduzida, como a encontrada em pacientes renais crônicos ${ }^{26}$, hipertensos ${ }^{27}$, diabéticos ${ }^{28}$ e nos obesos participantes deste estudo, pode aumentar a suscetibilidade destes indivíduos a danos oxidativos e, em sentido contrário, o aumento dos níveis de GSH, redução do GSSG e diminuição do IE, observados após a operação, possivelmente reduz a incidência de lesões oxidativas e doenças relacionadas.

O TRAP fornece estimativa global das moléculas antioxidantes presentes em fluidos biológicos. Diversos modelos experimentais mostram aumento da geração de EROs e redução dos valores de TRAP ${ }^{29}$. Os níveis de TRAP do grupo obeso, no período pré-operatório, apresentavamse significativamente reduzidos em relação ao grupo controle. A partir do segundo mês da operação, observou-se aumento desta variável, e após 12 meses os pacientes não apresentavam diferença em relação ao grupo controle. Após a GVBYR, o aumento do TRAP possivelmente foi resultante da somatória de fatores observados devido à diminuição do peso, ou seja, redução dos níveis de marcadores inflamatórios, redução dos indicadores de lesão oxidativa e maior atividade dos marcadores relacionados à defesa antioxidante.

Em conclusão: a) no período pré-operatório, os indivíduos obesos apresentaram maior nível de indicadores de oxidação e menores índices de defesa antioxidante, ou seja, tiveram maior grau de estresse oxidativo; b) 12 meses após a operação, os níveis de indicadores de oxidação, apresentam redução significativa; c) houve redução significativa no nível de estresse oxidativo em 12 meses após a operação; d) o maior grau de proteção antioxidante observado após a operação, aliado à redução dos indicadores inflamatórios e de lesão oxidativa, indicaram menor grau de estresse oxidativo e fortaleceram o papel do tecido adiposo como agente de inflamação crônica.

\section{A B S T R A C T}

Objective: To evaluate the oxidative stress and inflammatory markers in obese patients before and after Roux-en-Y vertical banded gastroplasty. Methods: We studied 20 class III obese patients undergoing Roux-en-Y vertical banded gastroplasty, seven men and 13 women, mean age 39 years, and 20 non-obese subjects, nine males and 11 females, mean age 38 years. We determined the plasma levels of malondialdehyde, stress an index, total antioxidant capacity, catalase, reduced and oxidized glutathione and inflammatory markers (C reactive protein and á-1 acid glycoprotein). In the obese group, these parameters were determined before and 2, 6 and 12 months after gastroplasty. Results: Preoperatively, the obese group showed elevated levels of inflammatory markers of oxidative stress (malondialdehyde levels and stress index) and lower levels of indicators of antioxidant defense compared to the control group. Weight loss was accompanied by gradual reduction in the levels of malondialdehyde and stress index. We found an increased concentration of reduced glutathione and total antioxidant status and reduced levels of inflammatory markers. Conclusion: Weight loss improves the inflammatory state and oxidative stress levels.

Key words: Bariatric Surgery. Oxidative stress. Obesity. Biological markers.

\section{REFERÊNCIAS}

1. Furukawa S, Fujita T, Shimabukuro M, Iwaki M, Yamada M, Nakajima $Y$, et al. Increased oxidative stress in obesity and its impact on metabolic syndrome. J Clin Invest 2004; 114(12):175261.

2. Perticone F, Ceravolo R, Candigliota M, Ventura G, lacopino S, Sinopoli $F$, et al. Obesity and body fat distribution induce endothelia dysfunction by oxidative stress: protective effect of vitamin C. Diabetes 2001; 50(1):159-65

3. Dandona P, Mohanty P, Ghanim H, Aljada A, Browne R, Hamouda $W$, et al. The suppressive effect of dietary restriction and weight loss in the obese on the generation of reactive oxygen species by leukocytes, lipid peroxidation, and protein carbonylation. J Clin Endocrinol Metab 2001; 86(1):355-62.

4. Ceriello A, Motz E. Is oxidative stress the pathogenic mechanism underlying insulin resistance, diabetes, and cardiovascular disease ? The common soil hypothesis revisited. Arteriosc Thromb Vasc Biol 2004; 24(5):816-23.
5. Dixon $J B, O^{\prime} B r i e n ~ P E$. Lipid profile in the severely obese: changes with weight loss after lap-band surgery. Obes Res 2002; 10(9):90310

6. Selvin E, Paynter NP, Erlinger TP. The effect of weight loss on Creactive protein: a systematic review. Arch Intern Med 2007; 167(1):31-9

7. Mohamed-Ali V, Flower L, Sethi J, Hotamisligil G, Gray R, Humphries $\mathrm{SE}$, et al. beta-Adrenergic regulation of IL-6 release from adipose tissue: in vivo and In vitro. J Clin Endocrinol Metab 2001; 86(12): 5864-9.

8. Pasceri V, Willerson JT, Yeh ET. Direct proinflammatory effect of C-reactive protein on human endothelial cells. Circulation 2000; 102(18):2165-8

9. Prasad K. C-reactive protein increases oxygen radical generation by neutrophils. J Cardiovasc Pharmacol Ther 2004; 9(3):203-9.

10. Fournier T, Medjoubi-N N, Porquet D. Alpha-1-acid glycoprotein Biochim Biophys Acta 2000; 1482(1-2):157-71.

11. Yildirim A, Meral M, Kaybar H, Polat H, Ucar EY. Relationship between serum levels of some acute-phase proteins and stage of 
disease and performance status in patients with lung cancer. Med Sci Monit 2007; 13(4):CR195-200.

12. Gabay C, Kushner I. Acute-phase proteins and other systemic responses to inflammation. N Engl J Med 1999; 340(6):448-54.

13. Vannuchi $H$, Moreira EAM, Cunha DF, Junqueira-Franco MVM, Bernardes MM, Jordão Júnior AA. Papel dos nutrientes na peroxidação lipídica e no sistema de defesa antioxidante. Medicina 1998; 31(1):31-44.

14. Smith AD, Brands MW, Wang MH, Dorrance AM. Obesity-induced hypertension develops in young rats independently of the reninangiotensin-aldosterone system. Exp Biol Med 2006; 231(3):2827.

15. Palmieri VO, Grattagliano I, Portincasa P, Palasciano G. Systemic oxidative alterations are associated with visceral adiposity and liver steatosis in patients with metabolic syndrome. J Nutr 2006; 136(12):3022-6

16. Ribeiro SMR, Queiroz JH, Pelúzio MCG, Costa NMB, Matta SLP, Queiroz MELR. A formação e os efeitos das espécies reativas de oxigênio no meio biológico. Biosci J 2005; 21(3):133-49.

17. Dalle-Donne I, Rossi R, Colombo R, Giustarini D, Milzani A. Biomarkers of oxidative damage in human disease. Clin Chem 2006; 52(4):601-23

18. Loureiro APM, Di Mascio P, Medeiros MHG. Formação de adutos exocíclicos com bases de DNA: implicações em mutagênese e carcinogênese. Quim Nova 2002; 25(5):777-93.

19. Yesilbursa D, Serdar Z, Serdar A, Sarac M, Coskun S, Jale C. Lipid peroxides in obese patients and effects of weight loss with orlistat on lipid peroxides levels. Int J Obes 2005; 29(1):142-5.

20. Rogers RJ, Monnier JM, Nick HS. Tumor necrosis factor-alpha selectively induces MnSOD expression via mitochondria-to-nucleus signaling, whereas interleukin-1beta utilizes an alternative pathway. J Biol Chem 2001; 276(23):20419-27.

21. Dougall WC, Nick HS. Manganese superoxide dismutase: a hepatic acute phase protein regulated by interleukin- 6 and glucocorticoids. Endocrinology 1991; 129(5):2376-84.

22. Madamanchi NR, Vendrov A, Runge MS. Oxidative stress and vascular disease. Arterioscler Thromb Vasc Biol 2005; 25(1):29-38.
23. Sigfrid LA, Cunningham JM, Beeharry N, Lortz S, Tiedge M, Lenzen $S$, et al. Cytokines and nitric oxide inhibit the enzyme activity of catalase but not its protein or mRNA expression in insulin-producing cells. J Mol Endocrinol 2003; 31(3):509-18.

24. Meilhac O, Zhou M, Santanam N, Parthasarathi S. Lipid peroxides induce expression of catalase in cultured vascular cells. J Lipi Res 2000; 41(8):1205-13.

25. Storey KB. Oxidative stress: animal adaptations in nature. Braz J Med Biol Res 1996; 29(12):1715-33.

26. Durak I, Kavatcu M, Çimen MYB, Avci A, Elgün S, Öztürk HS. Oxidant/antioxidant status of erythrocytes from patients with chronic renal failure: effects of hemodialysis. Med Principles Pract 2001; 10(4):187-90. doi: 10.1159/000050367.

27. Redón J, Oliva MR, Tormos C, Giner V, Chaves J, Iradi A, et al. Antioxidant activities and oxidative stress by products in human hypertension. Hypertension 2003; 41(5):1096-101.

28. Darmaun D, Smith SD, Sweeren S, Sager BK, Welch S, Mauras N. Evidence for accelerated rates of glutathione utilization and glutathione depletion in adolescents with poorly controlled type 1 diabetes. Diabetes 2005; 54(1):190-6.

29. Salomão AB, Aguilar-Nascimento JE, Percario S, Sano V, Marques NR, Dias CCGO. Injeção na luz intestinal de glutamina aumenta a capacidade antioxidante total em equivalência ao trolox (TEAC) na isquemia-reperfusão hepática. Acta Cir Bras 2006; 21(4):6973.

Recebido em 10/05/2010

Aceito para publicação em 12/07/2010

Conflito de interesse: nenhum

Fonte de financiamento: nenhuma

\section{Como citar este artigo:}

Valezi AC, Cabrera EJ, Delfino VCA, Barbosa DS, Mali Júnior J, Menezes MA. Derivação gástrica em y-de-roux e a atividade inflamatória do tecido adiposo. Rev Col Bras Cir. [periódico na Internet] 2011; 38(3). Disponível em URL: http://www.scielo.br/rcbc

\section{Endereço para correspondência:}

Antônio Carlos Valezi

E-mail:valezi@sercomtel.com.br 